\title{
Analysis of Electromagnetic Interference Effect of the Pulse Interference on the Navigation Receiver
}

\author{
Xin Huang $\mathbb{D}$, Yuming Wang $\mathbb{D}$, and Yazhou Chen $(\mathbb{D}$ \\ Army Engineering University, Shijiazhuang Campus, National Key Laboratory on Electromagnetic Environment Effects, \\ Shijiazhuang 050000, China \\ Correspondence should be addressed to Yazhou Chen; chen_yazhou@sina.com
}

Received 27 September 2021; Revised 8 December 2021; Accepted 22 December 2021; Published 21 January 2022

Academic Editor: Angelo Liseno

Copyright (c) 2022 Xin Huang et al. This is an open access article distributed under the Creative Commons Attribution License, which permits unrestricted use, distribution, and reproduction in any medium, provided the original work is properly cited.

Aiming at the problem that satellite navigation signals are easily interfered by the radio frequency (RF) pulse signal, the electromagnetic interference effect of RF pulse on the navigation receiver is studied in this paper. A mathematical model of the pulse interference signal is established, and we choose the bit error rate (BER) as an indicator of the quality of the BDS signal. It is found that the BER is proportional to the duty cycle of the pulse signal and inversely proportional to the equivalent carrier-tonoise ratio $\left(\mathrm{C} / \mathrm{N}_{0}\right)$ through simulation. Then, an experiment of electromagnetic injection on the BDS receiver has been carried out, which studied the influence of the pulse interference parameters, such as repetition frequency and duty cycle on the $\mathrm{C} / \mathrm{N}_{0}$ of the BDS signal and the electromagnetic sensitivity threshold of the receiver. The comparative experiment between the pulse interference and the single-frequency continuous wave (CW) interference was also carried out, and we found that the effect of the pulse interference is better than that of single-frequency CW interference. The former is the correlation interference, and the latter is the blocking interference. Combined with the experiment phenomenon, the interference mechanism was further analyzed according to the relationship between the pulse period and the ranging code period of the navigation signal.

\section{Introduction}

In recent years, the Beidou Navigation Satellite System (BDS) independently developed by China has been widely used in military, civilian, and commercial fields. However, with the increasing development of electronic information equipment, the electromagnetic environment is full of intricate radio interference signals. Once the RF interference from frequency equipment or space radiation falls within the working bandwidth of the BDS receiver, it will seriously affect the acquisition and tracking performance of the receiver, reduce positioning accuracy, and even cause the satellite tracking loss [1-3].

The current researches mainly focus on the influence of $\mathrm{RF}$ interference on the quality of the navigation signal and the performance of acquisition and tracking [4-10]. In [11], through establishing the models of electromagnetic interference signals, the analytical formulas of the correlator output signal with different interference signals are deduced from the theoretical level and then concluding that the CW interference has the highest effect on the GPS receiver correlator compared with the narrowband interference, wideband interference, partial band interference, and so on. Researchers find that the interference effect of $\mathrm{CW}$ interference at different frequency points is affected by the frequency point, intensity, and receiver loop parameters [12]. In [13], the tracking error envelope of the coherent delay phase-locked loop is derived, and the performance of the receiver under the combined action of multipath interferences and CW interferences is studied, which concludes that its comprehensive influence depends on correlator spacing, interference signal frequency, reflected signal delay, and other factors. The above documents are mainly based on theory and simulation analyses and have a significant reference for the research of navigation system performance under interferences.

The electromagnetic environment effects of navigation receivers under the single-frequency $\mathrm{CW}$ interference were 
mainly studied in the previous researches. The CW interference has a single frequency, while the signal spectrum of the sine wave with the external square wave pulse modulation is composed of the fundamental wave and the harmonics. The higher harmonic components of the pulse interference signal spectrum will increase, which will theoretically increase the signal interference capability. In addition, there are pieces of literature that have shown that injected pulse signals with different pulse parameters will have different interference effects on the receiver. Studying the impact of pulse interference is an important basis for improving the safety and reliability of the navigation system [14-16]. In recent years, researches on pulse interference have mainly focused on theoretical analysis and software receiver simulation, which studied the impact of the pulse key parameters on the performance of the navigation receiver acquisition and tracking loop [17-20]. In [21], it takes the direct spread spectrum system as the research object and concludes that the pulse repetition frequency and pulse width have an important influence on the BER of the DS system. The BER is proportional to the interference power and the pulse width and inversely proportional to the pulse repetition frequency. In [22], the BER and $\mathrm{C} / \mathrm{N}_{0}$ of the receiving system with or without AGC are compared, and then the suppression effect of the RF front-end AGC on the pulse interference is studied.

In addition to the theoretical analysis, there are also some researchers to study the interference effects of GNSS by the electromagnetic experiment [23-26]. Literature [27] uses the method of parallel code phase capture and simulates the capture performance of the receiver under single-frequency interference based on Simulink and verifies the equivalence of the two through radiation and injection electromagnetic effects experiments. Literature [28] takes a certain type of navigation receiver as the experiment object, carries out the CW injection effect experiment, obtains the electromagnetic sensitivity threshold curve of different satellites, finds the electromagnetic sensitive frequency band under the singlefrequency interference of this type of receiver, and proposes feasible electromagnetic interference prediction method.

Based on the above previous researches, this paper mainly emphasizes the BDS receiver of the B1I signal. The main contributions are described as follows:

(1) In Section 1, we give the waveform of the pulse interference and analyze the impact of the pulse interference on the demodulation performance of the navigation receiver

(2) In Section 2, a certain BDS receiver is taken as the research object to carry out the pulse interference injection experiments, focusing on investigating the positioning function of the receiver under the influence of the pulse interference with different interference powers, repetition frequencies, and duty cycles

(3) In Section 3, combining the relationship between the spectrum characteristics of the pulse interference signal and the navigation ranging code, the mechanism of the pulse interference is analyzed, which supplements the study of electromagnetic interference effects of such RF interference on the navigation receiver

\section{Pulse Interference Analysis}

2.1. The Model of the Pulse Interference Signal. The pulse interference signal refers to an interference signal, which is composed of a series of narrow pulses repeated in the period. It should be noted that this kind of pulse interference is a RF pulse signal modulated by a sinusoidal carrier, which can be modeled in the time domain as [29]

$$
I_{P U I}(t)=p(t) \sqrt{2 J} \cos \left(2 \pi f_{i} t+\theta_{i}\right),
$$

where $J$ is the signal power of the pulse interference, $f_{i}$ is the carrier center frequency, $\theta_{i}$ is the initial phase, and $p(t)$ is the baseband pulse signal. Here is the square wave pulse, the time domain expression of which can be written as [29]

$$
p(t)=\left\{\begin{array}{l}
A, m T_{p} \leq t \leq(m+D) T_{p}, m=\text { int }, \\
0, \text { else }
\end{array}\right.
$$

where $A$ is the amplitude of the square wave signal and $T_{p}$ and $D$ are the pulse period and duty cycle of the pulse signal respectively, which are the two main parameters of the pulse interference, and the repetition frequency $f_{P R F}=1 / T_{p}$.

We can get the frequency spectrum of the pulse signal as (3) via the Fourier transform of (2):

$$
p(f)=D \sum_{k=-\infty}^{+\infty} \sin c(k \pi D) \delta\left(f-\frac{m}{T_{p}}\right) .
$$

It specifically shows that the spectral envelope of the baseband pulse signal is a sinc function, which is composed of discrete spectral lines with an interval of $1 / T_{p}$, and the main lobe bandwidth of the spectral line is $1 /\left(D T_{p}\right)$.

In the BDS signal, the frequency spectrum of the ranging code used for spread-spectrum modulation of navigation message data is also composed of discrete spectral lines [30], and the spectral line interval is $1 / T_{c}\left(T_{c}=1 \mathrm{~ms}\right.$, which is the period of the ranging code). The pulse interference has the same signal type and power spectrum form as the BDS ranging code. The certain similarity in the signal system makes it easy to overlap with navigation signals in the baseband demodulation process, thereby affecting the relevant processing of navigation signals.

\subsection{Impact of the Pulse Interference on the Demodulation} Performance. In the correlation processor, the pulse interference signal is multiplied by the local reference ranging code generated by the correlator, and then the integrator performs coherent integration processing within the integration time $T_{d}$. The final interference power output by the correlator is, 


$$
P_{P U I}=k\left|\frac{1}{N_{s}} \sum_{n=0}^{N_{s}-1} \sqrt{2 J} \sum_{z=1}^{\infty} p\left(n T_{s}-z T_{p}\right) \cdot c\left(n T_{s}-\tau_{n}\right) e^{j\left(2 \pi \Delta f_{i} n T_{s}+\Delta \theta_{i}\right)}\right|,
$$

where $c(t)$ is the locally generated reference spreading code, $\tau_{n}$ is the transmission delay, $\Delta f_{i}$ and $\Delta \theta_{i}$ are, respectively, the interference frequency and phase error. Formula (4) shows that the effect of the pulse interference signals on navigation signals is affected by the combination of pulse parameters, interference frequency offset, and ranging code characteristics.

The equivalent $\mathrm{C} / \mathrm{N}_{0}$ is the $\mathrm{C} / \mathrm{N}_{0}$ at the output of the correlator of the navigation receiver. In the presence of the interference, the interference power output by the correlator can be equivalent to the increment of the noise power. At this time, the equivalent $\mathrm{C} / \mathrm{N}_{0}$ under the pulse interference is,

$$
\left[C / N_{0}\right]_{e q}=\frac{P_{s}}{P_{N}+P_{P U I}},
$$

$$
P_{e}=(1-D) Q\left(\sqrt{\frac{2\left[C / N_{0}\right]_{e q}}{N_{0} R_{S}\left(P_{N}+P_{P U I}\right)}}\right)+D Q\left(\sqrt{\frac{2\left[C / N_{0}\right]_{e q}}{\left(N_{0}+N_{J}\right) R_{S}\left(P_{N}+P_{P U I}\right)}}\right) .
$$

It can be seen from (7) that the BER is inversely proportional to the equivalent $\mathrm{C} / \mathrm{N}_{0}$ and directly proportional to the duty cycle. Assuming that the signal-to-noise ratio (SNR) of the navigation signal is $10 \mathrm{~dB}$, when no interference is applied, we can get the simulation result of the BER under the pulse interference as shown in Figure 1.

It can be seen from Figure 1 that as the duty cycle of the pulse interference increases, the BER increases. When the jamming-to-signal ratio (JSR) of the pulse interference is $-20 \mathrm{~dB}$, the BER is extremely small and the pulse interference almost has no interference effect on the navigation system currently. With the gradual increase of JSR, the BER under the same duty cycle will increase accordingly. When JSR $>10 \mathrm{~dB}$, the increase of JSR has a little effect on the further degradation of the navigation system performance. It shows that the interference power of the pulse interference largely determines the interference effect, but when the interference power reaches a certain threshold, the BER of the system tends to remain unchanged with the increase of JSR. At this time, the BER is no longer the effective indicator of the quality of the navigation receiver. If we continue to increase the JSR, the pulse interference will have a serious impact on the acquisition and tracking link, resulting in the loss of loop lock and satellite tracking, which greatly affects the performance of the navigation system. where $P_{s}$ is the navigation signal power at the output of the correlator and $P_{N}$ is the noise power at the output of the correlator.

The navigation signal is modulated by BPSK. According to the best detection theory of the receiver, the BER of the system under the pulse interference is,

$$
P_{e}=(1-D) Q\left(\sqrt{\frac{2 E_{b}}{N_{0}}}\right)+D Q\left(\sqrt{\frac{2 E_{b}}{N_{0}+N_{J}}}\right),
$$

where $N_{0}$ is the unilateral power spectral density of noise, $E_{b}$ is the signal power carried by the signal per bit $\left(E_{b}=P_{s} / R_{s}\right)$, $N_{J}$ is the power spectral density of the pulse interference, and $R_{s}$ is the spreading code rate. By substituting $E_{b}$ into formula (6), we will have

\section{Electromagnetic Injection Effect Experiment of the Pulse Interference Signal}

To verify the reliability of the above theoretical analysis and simulation results, and explore the interference of navigation signals and the degradation of the BDS receiver performance under the actual pulse interference, this section is aimed at a certain BDS receiver, and the following electromagnetic injection experiments are carried out. The BDS signals that we investigate in this paper are the B1I signals, adopting BPSK modulation, and the working frequency of which is $1561 \mathrm{MHz}$.

3.1. Setup of the Experiment System. There are two common experimental methods to research the electromagnetic interference effects of the navigation receiver [25-28]. The irradiation method is to use a transmitting antenna to transmit the navigation signal and the interference signal in a specific microwave darkroom environment. Electromagnetic waves are radiated in space, and the navigation receiver receives them through the receiving antenna. The injection method is to directly inject the navigation signal and the interference signal into the radio frequency input port of the navigation receiver through the cable. The difference between the two experimental methods is that the way of 


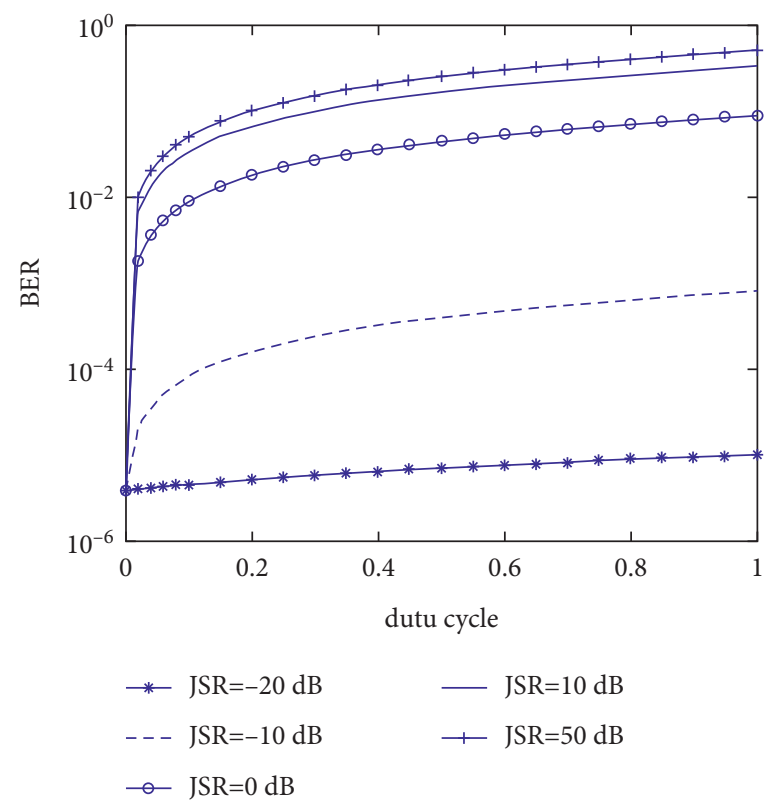

FIgURE 1: The simulation result of BER with pulse interference.

receiving the signals is different. The electromagnetic waves transmitted in the irradiation experiment will have some loss in the air. Moreover, it is greatly affected by environmental noise. The injection experiment will equivalently inject electromagnetic signals into the receiver, which can achieve the same interference effect, but the experiment has high repeatability and is less affected by the environment.

Based on the injection method, an electromagnetic experiment system for the BDS receiver under the pulse interference is designed, and the electromagnetic susceptibility of the BDS receiver under the different repetition frequencies and duty cycles is studied. In this section of the experiment, a square wave pulse externally modulated single-frequency $\mathrm{CW}$ is used to simulate the interference signal of the BDS receiver, and the detailed expression of the interference signal is shown in (1). The block diagram of the experiment configuration is shown in Figure 2, and the builtup electromagnetic injection experiment platform is shown in Figure 3.

The experiment system can be divided into two parts: the injection end and the receiving end.

The injection end includes two interference signal generators, a navigation signal simulator, an adjustable attenuator, and a combiner. Two different interference signal generators, respectively, generate a square wave pulse signal and a single-frequency $\mathrm{CW}$ signal. The square pulse signal modulates the CW signal, and then a RF pulse interference signal is output. Since the measured navigation signals of the satellites are affected by weather and venues. Using actual satellite collection data to carry out each experiment does not have repeatability and practical operability. Therefore, before the experiment, the receiving antenna and the BDS receiver are used to measure and record the number of satellites and corresponding $\mathrm{C} / \mathrm{N}_{0}$ in the experiment area. The experiment scenario is shown in Figure 4. On this basis, a navigation signal simulator is set up to broadcast satellite navigation signals consistent with the actual measurement data in the region, which are set as the BDS signals required for the experiment. The interference signal and the navigation signal are mixed by the combiner and then transmitted to the receiving end to realize the injection process of the mixed signals.

The receiving end is mainly composed of a RF front-end, a BDS receiver, and a monitoring computer. The mixedsignal injected through the combiner enters the RF frontend module for filtering, mixing, and low-noise amplifier processing and then is transmitted to the receiver for despreading and demodulating processing; finally, the original navigation message information is output. Both the navigation receiver and the navigation signal simulator are connected to the monitoring computer through data transmission lines. The $\mathrm{C} / \mathrm{N}_{0}$ of the broadcasting satellite, the positioning, and the tracking status of the BDS receiver are displayed in real time through the monitoring software. This type of BDS receiver adopts the four-star positioning principle and uses monitoring software to observe the changes in the $\mathrm{C} / \mathrm{N}_{0}$ of each satellite at the current moment. When the $\mathrm{C} / \mathrm{N}_{0}$ of a single satellite is lower than the tracking threshold, the satellite will lose track. Once the number of trackable satellites is less than four, the receiver loses its navigation and positioning function. At this time, the interference signal power is the electromagnetic sensitivity threshold of the BDS receiver.

3.2. Experiment Procedure. According to the experiment configuration shown in Figure 2, an experiment platform for electromagnetic injection of the pulse interference on the BDS receiver is set up. Since the landing level of the navigation signal is about $-160 \mathrm{dBW}$, which is pretty susceptible to the interference signal of lower power, so it is necessary to add an attenuator at the injection end, and the attenuation 


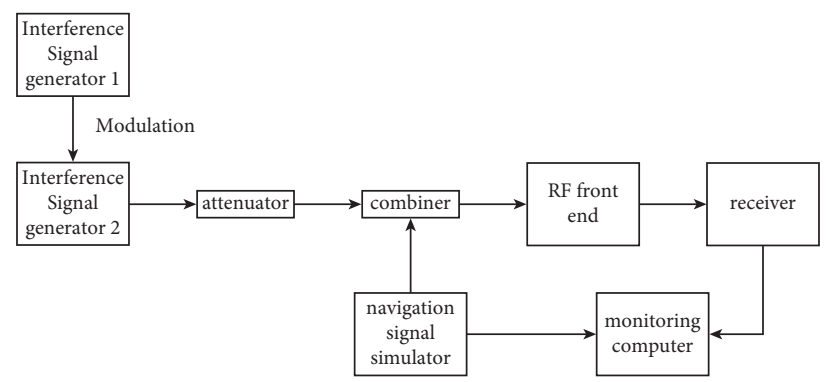

FIGURE 2: Block diagram of experiment configuration.

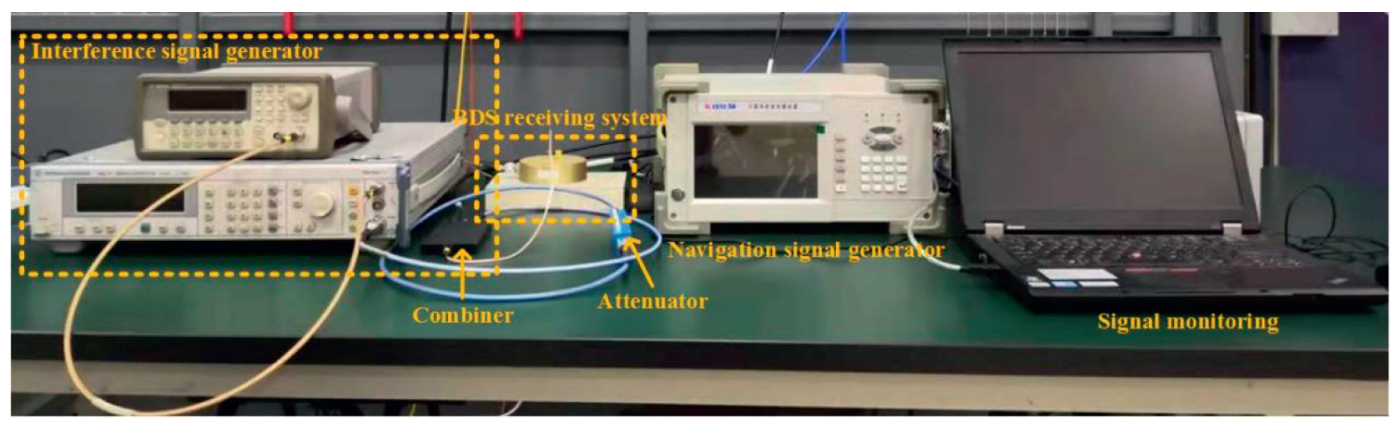

FIGURE 3: Electromagnetic injection effect experiment platform of the pulse interference.

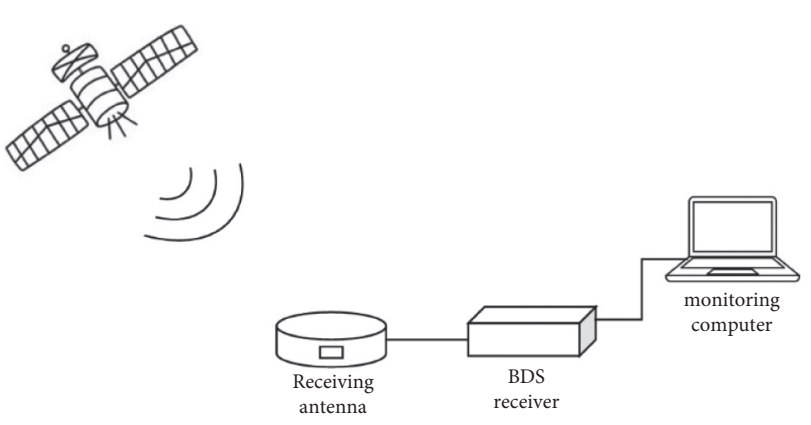

Figure 4: Actual satellite experiment scenario.

multiple is set to $60 \mathrm{~dB}$. Adjust the output power of the navigation signal simulator according to the actual situation of receiving satellites in the early stage and carry out injection experiments on this basis. The initial $\mathrm{C} / \mathrm{N}_{0}$ of the actual tracking satellite is shown in Table 1, and the sky plot of the actual satellites is shown in Figure 5.

To measure the electromagnetic interference effect of the pulse interference on BDS, the change of the $\mathrm{C} / \mathrm{N}_{0}$ of a single satellite and whether the receiver is accurately positioned are used as evaluation indicators. Therefore, the experiment is mainly divided into the following two aspects.

\subsubsection{Single Satellite C/NO Changes with the Interference} Power. After preliminary experiments, when the interference frequency is set to $1561 \mathrm{MHz}$ and the output interference power is less than $-88 \mathrm{dBm}$, the interference effect is not significant; meanwhile, the satellite $\mathrm{C} / \mathrm{N}_{0}$ is basically not affected. Therefore, the interference power is set to gradually increase by $3 \mathrm{~dB}$ from $-88 \mathrm{dBm}$ with each power state lasting
TABle 1: Tracking satellite initial $\mathrm{C} / \mathrm{N}_{0}$.

\begin{tabular}{lcccccc}
\hline Satellites number & 1 & 3 & 6 & 8 & 9 & 12 \\
\hline Initial $\mathrm{C} / \mathrm{N}_{0}(\mathrm{~dB} \bullet \mathrm{Hz})$ & 35 & 40 & 40 & 43 & 37 & 38 \\
\hline
\end{tabular}

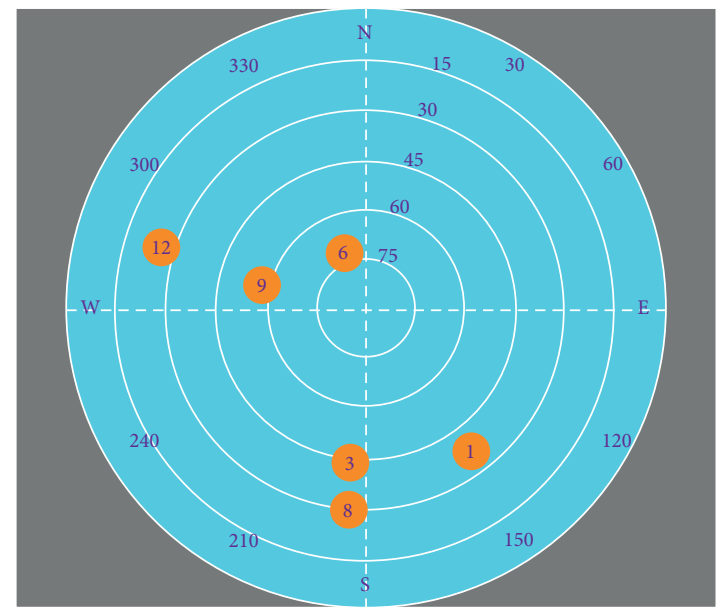

FIGURE 5: The sky plot of the satellites.

for $10 \mathrm{~s}$, and the real-time $\mathrm{C} / \mathrm{N}_{0}$ value under each power value is recorded until the satellite tracking is lost.

\subsubsection{The BDS Receiver Positioning Loss Sensitivity} Threshold. The interference signal generator 1 is used to generate the baseband pulse signal, and the parameter settings of the signal are shown in Table 2. The interference signal generator 2 generates a single-frequency CW. Under 
TABle 2: Pulse signal parameter setting.

\begin{tabular}{lccc}
\hline Parameter & Peak voltage & Repetition frequency & Duty cycle \\
\hline Value & $3 \mathrm{vpp}$ & $60 \mathrm{~Hz}, 1 \mathrm{kHz}, 1 \mathrm{MHz}$ & $40 \%, 60 \%, 80 \%$
\end{tabular}

the premise of determining the repetition frequency and duty cycle, the center frequency of the $\mathrm{CW}$ is adjusted to make it uniformly change within the BDS signal bandwidth (1559 MHz-1563 MHz), with a step of $0.2 \mathrm{MHz}$. The power of the interference signal is kept increasing until the number of satellites tracked by the BDS receiver is less than four, and the positioning is out of lock state. Then, the interference power at this time is recorded as the sensitivity threshold at this frequency. After obtaining the sensitivity threshold of the BDS receiver under different pulse parameters, the electromagnetic sensitivity curves can be drawn.

\subsection{Experiment Results and Analysis}

3.3.1. The $C / N_{0}$ of a Single Satellite. According to the abovementioned experiment method, with the No. 3 satellite as the experiment target, the changes of the satellite $\mathrm{C} / \mathrm{N}_{0}$ under different pulse parameters are obtained, which are shown in Figure 6.

It can be seen from Figure 6 that different pulse parameters will significantly affect the change of the $\mathrm{C} / \mathrm{N}_{0}$ of the No.3 satellite. Under the same conditions of the repetition frequency and the interference power, the interference effect of the high duty cycle is obviously better than that of the low duty cycle. When the interference power is small, the duty cycle has no obvious influence on the $\mathrm{C} / \mathrm{N}_{0}$. With the increase of the interference power, the difference of the $\mathrm{C} / \mathrm{N}_{0}$ under the interference of different duty cycles gradually increases. Under the condition of the same duty cycle of $60 \%, 1 \mathrm{MHz}$ pulse interference has the worst effect, which shows that increasing the repetition frequency can effectively suppress the effect of interference on the signal $\mathrm{C} / \mathrm{N}_{0}$.

To further study the influence of the pulse interference on the variation of satellite $\mathrm{C} / \mathrm{N}_{0}$ under different conditions, the following two comparative experiments are carried out.

(1) The Influence of Satellite Type on the Change Rule of Satellite $C / N_{0}$. To realize global autonomous navigation, positioning, short message communication, and other services, the Beidou system uses 35 satellites to form the space constellation part, of which 5 are experimental satellites, and the remaining 30 are nominal satellites, specifically 24 medium circular orbit satellites (MEO) +3 geostationary orbit satellites (GEO) + 3 Earth tilted orbit satellites (IGSO). The GEO satellite broadcasts the D2 navigation message, while the MEO/IGSO satellite broadcasts the D1 navigation message. There is a difference in the navigation signal system between the two [31-33]. The No.3 satellite is a GEO satellite, and the No.6 satellite is an MEO/IGSO satellite. To explore the interference law of pulse interference to different types of BDS signals, the No.6 satellite is now selected as the experiment object to carry out a comparative experiment. According to the above method, the different pulse

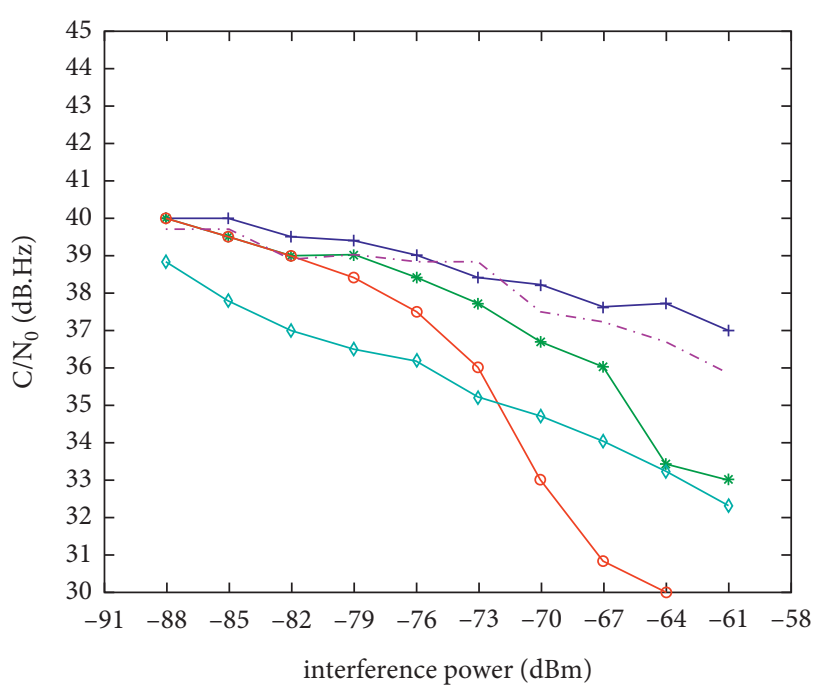

$$
\begin{aligned}
& \text { — } 1 \mathrm{KHz} 40 \% \text { ○ } 60 \mathrm{~Hz} 60 \% \\
& \text { * }-1 \mathrm{KHz} 60 \% \\
& \text { ○- } 1 \mathrm{KHz} 80 \%
\end{aligned}
$$

Figure 6: The $\mathrm{C} / \mathrm{N}_{0}$ in different pulse parameters (PRN3).

parameters are measured. The change rule of $\mathrm{C} / \mathrm{N}_{0}$ is shown in Figure 7.

Comparing Figures 6 and 7, we can see that when the pulse interference repetition frequency is $1 \mathrm{kHz}$, the $\mathrm{C} / \mathrm{N}_{0}$ of the No.3 and No.6 satellites are basically the same under the same interference power, indicating the difference in ranging code sequences does not affect the effect of the pulse interference with a repetition frequency of $1 \mathrm{kHz}$. The changing trend of the $\mathrm{C} / \mathrm{N}_{0}$ of different satellites is basically the same under the condition of $1 \mathrm{MHz}$. The change of the $\mathrm{C} / \mathrm{N}_{0}$ of different satellites under the condition of $60 \mathrm{~Hz}$ is quite different. The $\mathrm{C} / \mathrm{N}_{0}$ of the No.3 satellite is reduced to $32 \mathrm{~dB} \cdot \mathrm{Hz}$ which is the tracking threshold when the interference power is $-61 \mathrm{dBm}$, while the No.6 satellite has lost track when the interference power is just $-77 \mathrm{dBm}$.

The external modulation of the square wave pulse is removed, and the interference signal is directly injected into the $\mathrm{BDS}$ receiver in the form of a single frequency $\mathrm{CW}$. In the meantime, the center frequency of the interference signal is still set to $1561 \mathrm{MHz}$. Through the experiment, we find that when the interference intensity is lower than $-60 \mathrm{dBm}$, the C/N $\mathrm{N}_{0}$ of satellites No.3 and No. 6 basically does not change, and it fluctuates within the range of $1 \mathrm{~dB}$ up and down $40 \mathrm{~dB} \cdot \mathrm{Hz}$. It is specifically shown that the interference signal type has a greater impact on the BDS receiver when the interference frequency is $1561 \mathrm{MHz}$. The interference effect of the pulse signal is significantly stronger than the singlefrequency $\mathrm{CW}$ interference, which poses a greater threat to the navigation and positioning function of the $\mathrm{BDS}$ receiver.

(2) The Influence of the Initial $C / N_{0}$ on the Change Rule of the Satellite $C / N_{0}$. Satellites No. 1 and No. 3 are both GEO satellites and have the same signal type. The initial $\mathrm{C} / \mathrm{N}_{0}$ are $35 \mathrm{~dB} \cdot \mathrm{Hz}$ and $40 \mathrm{~dB} \cdot \mathrm{Hz}$, respectively. Figure 8 shows the 


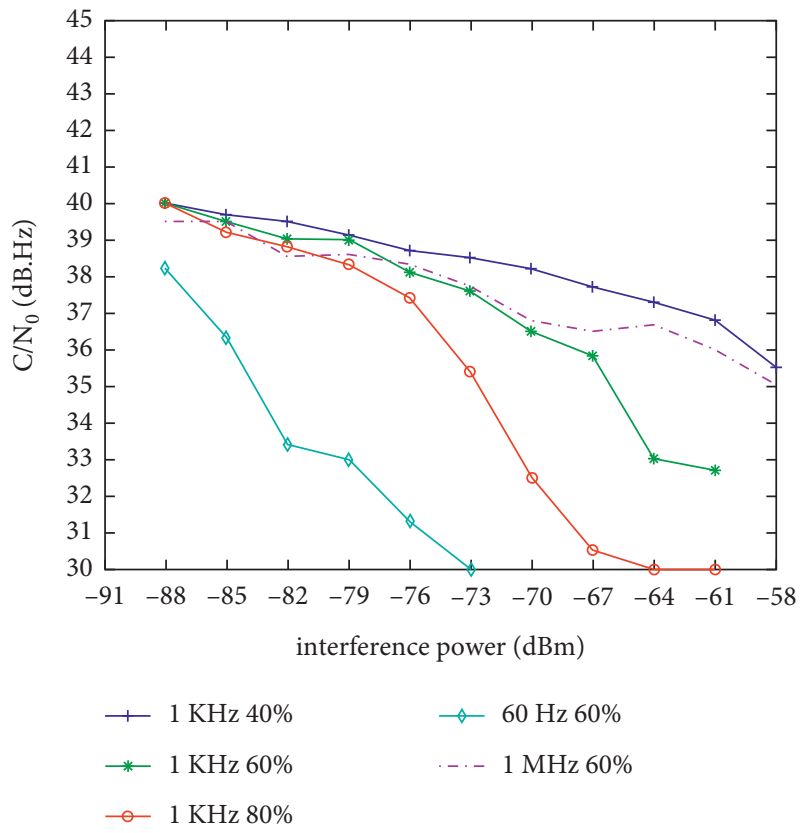

Figure 7: $\mathrm{C} / \mathrm{N}_{0}$ in different pulse parameters (PRN6).

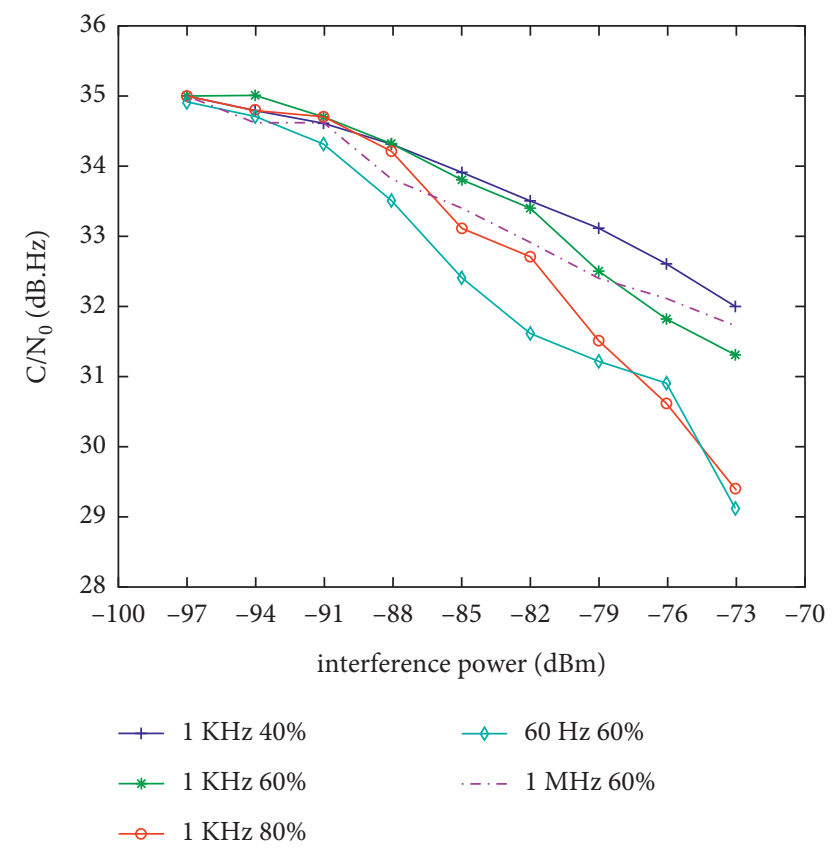

Figure 8: $\mathrm{C} / \mathrm{N}_{0}$ in different pulse parameters (PRN1).

change of $\mathrm{C} / \mathrm{N}_{0}$ of satellite No. 1 under the interference of different pulse parameters.

Comparing Figures 6 and 8, we can see that the changing trend of the satellite real-time $\mathrm{C} / \mathrm{N}_{0}$ is basically the same in the case of low initial $\mathrm{C} / \mathrm{N}_{0}$ and that of high initial $\mathrm{C} / \mathrm{N}_{0}$. When the initial $\mathrm{C} / \mathrm{N}_{0}$ is low, the interference power imposed to cause the satellite $\mathrm{C} / \mathrm{N}_{0}$ falling to the tracking threshold is low, and the navigation signal is more susceptible to the interference.
3.3.2. The Sensitivity Threshold of the BDS Receiver. According to the experiment method described in Section 3.2, the sensitivity threshold of the BDS receiver under different interference signal parameters is obtained. Figures 9 to -11 are the electromagnetic sensitivity curves of the receiver when the pulse signal repetition frequency is, respectively, $60 \mathrm{~Hz}, 1 \mathrm{kHz}$, and $1 \mathrm{MHz}$.

Figure 9 shows the electromagnetic sensitivity curve of the receiver when $T_{p}>T_{c}$. The sensitivity curves under different duty cycles change basically the same with the interference frequency, but the change of the duty cycle significantly affects the receiver sensitivity threshold, resulting in a maximum difference of $10 \mathrm{~dB}$ in the interference power with different duty cycles. As the duty cycle increases, the interference effect of the pulse interference on the navigation signal gradually increases. When the interference frequency is between $1561 \mathrm{MHz}$ and $1561.2 \mathrm{MHz}$, the receiver sensitivity curve has an obvious trough, which is the sensitive frequency band of the receiver under the pulse interference.

Figure 10 shows the electromagnetic sensitivity curve of the receiver when $T_{p}=T_{c}$. The change of the duty cycle causes the most sensitive frequency point in the navigation signal band to shift, which no longer overlaps with the worst spectral line frequency point under the single-frequency $\mathrm{CW}$ [34], and the optimal interference frequency point under different duty cycles is also not the same. We can see that the sensitivity threshold at $80 \%$ is lowest at $1561 \mathrm{MHz}$, which from $1560.8 \mathrm{MHz}$ to $1561 \mathrm{MHz}$ has decreased sharply by $3 \mathrm{~dB}$. The result of the analysis is that when the duty cycle is $80 \%$ and the interference frequency is $1561 \mathrm{MHz}$, the frequency difference between the interference spectral line falling into the correlator and the spectral line of the nearest ranging code is exactly zero, and the interference effect is the best, so the threshold has decreased significantly. This phenomenon has also appeared in the single-frequency $\mathrm{CW}$ interference experiment. Combined with Figures 9 and 10, the duty cycle has a greater impact on the sensitivity threshold of the BDS receiver in the case of low repetition frequency. In other words, the BDS signal is sensitive to the duty cycle.

Figure 11 shows the electromagnetic sensitivity curve of the receiver when $T_{p}<T_{c}$. The sensitivity of BDS signals to the duty cycle is quite different from that in low repetition conditions. The sensitivity threshold at $40 \%$ is between $60 \%$ and $80 \%$. This result can be explained by the fact that a decrease in the duty cycle means an increase in the main lobe bandwidth of the spectral line, increasing the possibility of more sinc tails coinciding with the sinc function resulting from the integrator in the tracking loop. Meanwhile, there are also measurement errors coming from the instruments. Taking these factors into account, we can intuitively see that the sensitivity thresholds under different duty cycles are not much different; because the repetition frequency of the pulse interference is very high, only one interference spectrum line falls within the ranging code envelope. The pulse interference is equivalent to the single-frequency $\mathrm{CW}$, so the electromagnetic sensitivity curve is similar to that of the single-frequency $\mathrm{CW}$, which accounts for that the receiver is 


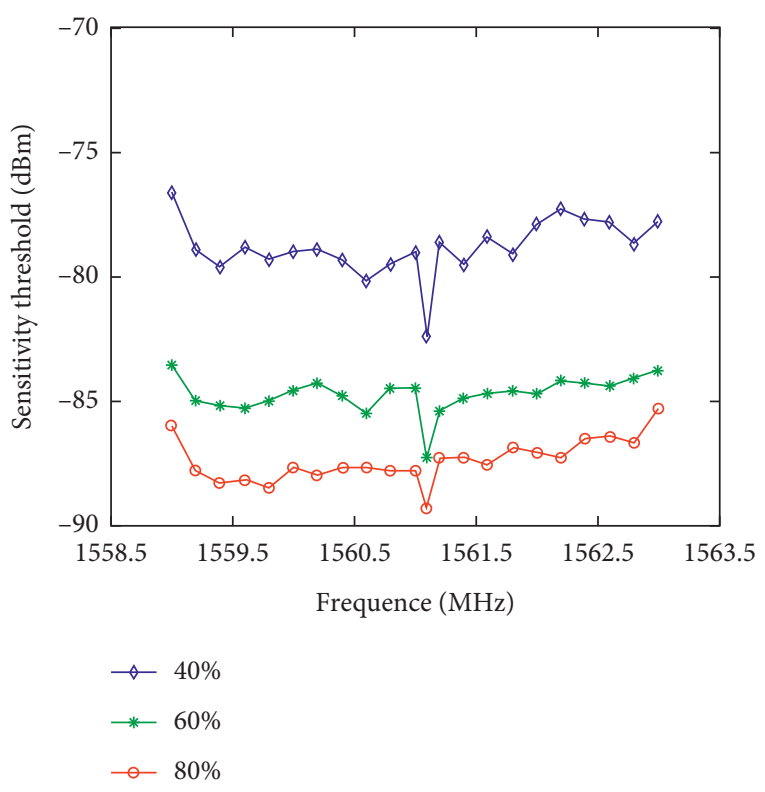

FIGURE 9: The electromagnetic sensitivity curve of the receiver when $f_{P R F}=60 \mathrm{~Hz}$.

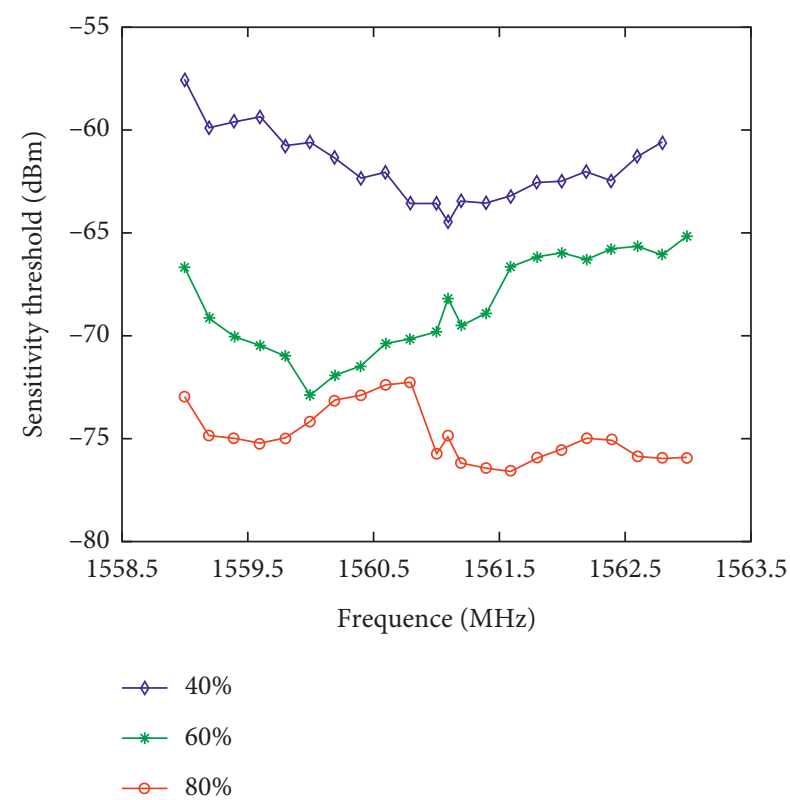

FIgURE 10: The electromagnetic sensitivity curve of the receiver when $f_{P R F}=1 \mathrm{kHz}$.

not sensitive with duty cycle when the repetition frequency is very high.

Figure 12 is a comparison of the sensitivity threshold curves of three different repetition frequencies to the receiver under the condition of $60 \%$ duty cycle. It vividly shows that when the duty cycle is constant, as the repetition frequency increases, the receiver sensitivity threshold increases, and the pulse interference performance decreases, which is because the increase in repetition frequency means that the pulse period becomes shorter, leading to the sparse interval of the discrete spectral lines, and the number of the

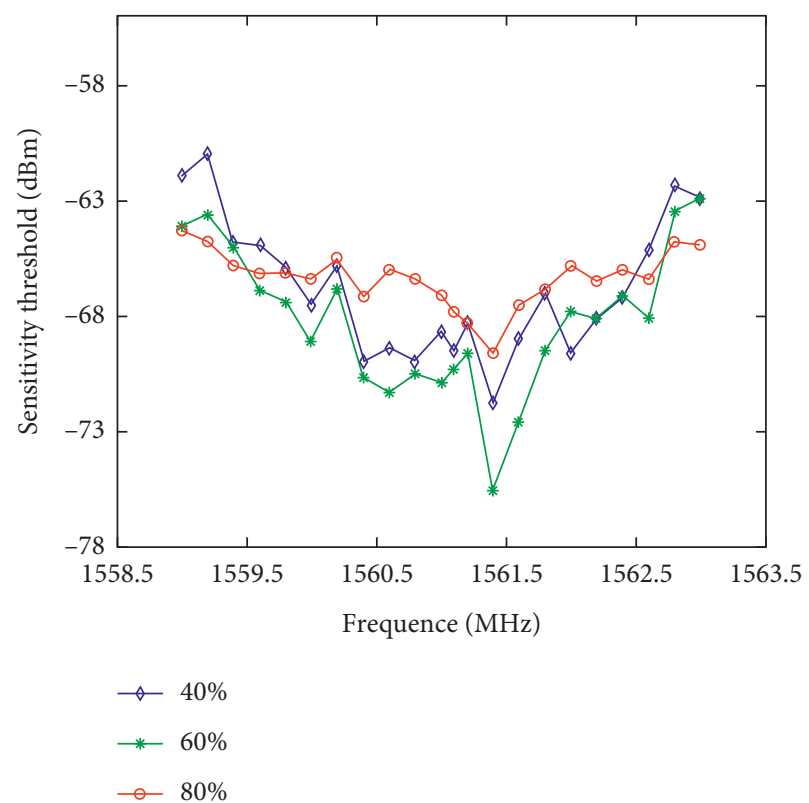

FIGURE 11: The electromagnetic sensitivity curve of the receiver when $f_{P R F}=1 \mathrm{MHz}$.

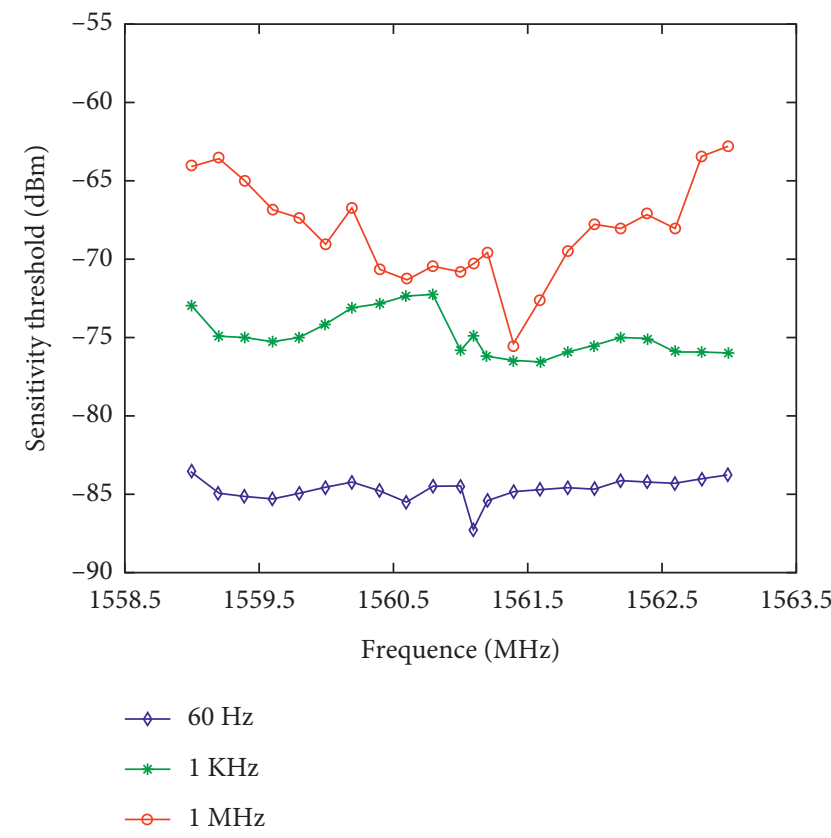

FIGURE 12: The electromagnetic sensitivity curve of the receiver under $60 \%$ duty cycle.

pulse spectral lines in a certain frequency domain also decreases. The energy of the interference signal entering the correlator decreases and the average interference power decreases, so the interference effect is weakened. Consequently, an appropriate increase in the pulse repetition frequency can achieve the purpose of suppressing pulse interference and provide an idea for weakening the impact of the pulse interference.

Based on the overall analysis of Figures 9 12, it can be seen that the BDS signal is more sensitive to the repetition 
frequency of the pulse interference signal. The duty cycle has little impact on the sensibility of the BDS signal and the most sensitive frequency point position under the condition of high repetition frequency, while it is easy to affect the sensitivity threshold of the receiver in the case of low repetition frequency, and the difference can reach up to $15 \mathrm{~dB}$.

\section{The Analysis of the Pulse Interference Mechanism}

According to the pulse interference effects obtained in Section 3.3, the following two main conclusions can be drawn:

(1) From the perspective of the effect of electromagnetic interference on a single-channel navigation signal, when the interference frequency falls within the navigation signal band, the pulse interference can achieve an effective interference effect equivalent to single-frequency CW with less interference power

(2) The pulse repetition frequency has a greater impact on the interference effect, and the interference effect gradually becomes worse with the increase of the repetition frequency

The following is a specific analysis of the interference mechanism of the pulse interference to the navigation receiver for the above two points.

During the experiment, the IF output signal observed by the spectrum analyzer did not have obvious distortion, indicating that the signal received by the navigation receiver did not exceed the dynamic range of the RF front-end, and the gain given to the navigation signal by the AGC module was not compressed, so the impact location of pulse interference on the receiver was determined as the baseband digital processing part. Because the signal form and power spectrum of the pulse interference are correlated with the ranging code of the navigation signal, under the same interference power, the pulse interference has a greater chance of locating in the position of the ranging code spectrum line compared with the CW interference, which causes the interference effect is strengthened. The interference effect of single-frequency CW interference is maximized only when the frequency deviation is aligned with the position of the worst spectral line. Therefore, the interference effect of the pulse interference is better than that of the single-frequency CW.

After the pulse interference reaches the carrier tracking loop through the prefilter, it is first multiplied by the locally generated carrier sequence to complete the carrier stripping, and then the code stripping is realized by the code correlator processing; finally, the coherent integration operation is performed. Due to the presence of the interference signals, the loop noise will increase, and the $\mathrm{C} / \mathrm{N}_{0}$ of the output signal of the correlator decreases, which affects the output result of the correlator. Formula (8) is the frequency domain expression of the mixed signal of the pulse interference signal and the navigation signal after being processed by the correlator, and the effect of Gaussian white noise is not considered here:

$$
Z(f)=\sum_{m} \sum_{k} T_{d} J_{n} P_{m} C_{k} \times \operatorname{sinc}\left(\left(f-\frac{n-k}{T_{c}}+\frac{m}{T_{p}}-\Delta f_{i}\right) T_{d}\right) e^{-j 2 k \pi \tau / T_{c}},
$$

where $T_{d}$ is the integration duration, $J_{n}$ is the interference signal power, $P_{m}$ and $C_{k}$ are the spectral line coefficients of the pulse discrete spectral line and the ranging code discrete spectral line, and $\tau$ is the phase difference of the reference code. To further simplify formula (8), the discussion needs to be divided into the following three situations.
(1) $T_{p}>T_{c}$. Figure 13 shows the sinc function envelope relationship between the pulse interference spectrum line and the ranging code spectrum line under the condition of $T_{p}>T_{c}$. The ranging code envelope can cover most of the pulse function envelope, which can be inferred that the terms in (8) with $n \neq \mathrm{k}$ are zero, so the output frequency domain signal of the correlator under this condition is

$$
Z(f)=\sum_{m=-M}^{+M} T_{d} J_{n} P_{m} C_{n} \times \operatorname{sinc}\left(\left(f+\frac{m}{T_{p}}-\Delta f_{i}\right) T_{d}\right) e^{-j 2 n \pi \tau / T_{c}},
$$

where $M=T_{p} / T_{d}$. It can be seen from Figure 13 that when $T_{p}>T_{c}$, the pulse interference has more energy that can enter the correlator and cause interference to the navigation signal. The main lobe width of the pulse interference spectrum is $1 /\left(D T_{p}\right)$, and the change of the duty cycle will cause the change of the width of the main lobe and then affect the degree of correlation between the pulse spectrum and the ranging code spectrum. Therefore, the interference energy which is the output of the correlator under this condition is sensitive to the duty cycle, which is consistent with the experiment results.

(2) $T_{p}=T_{c}$. Figure 14 shows the sinc function envelope relationship between the pulse interference spectrum line and the ranging code spectrum line under the condition of $T_{p}=T_{c}$. In the case of $T_{p}=T_{c}$, the spectral line interval of the pulse spectrum is equal to the navigation signal spectral line 


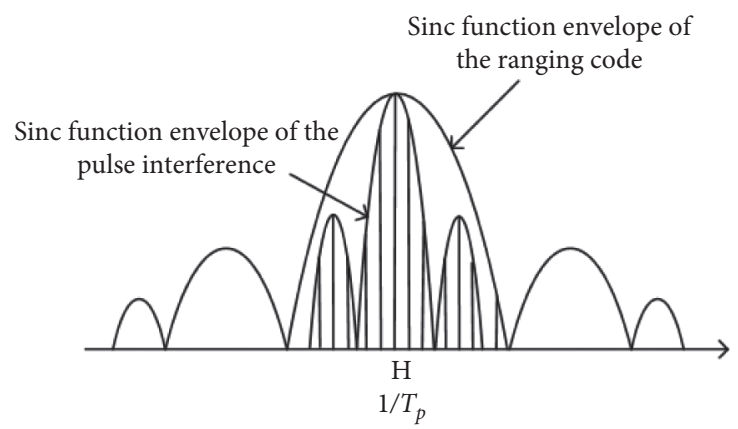

FIGURE 13: Sinc function envelope of the pulse interference and ranging code spectrum line $\left(T_{p}>T_{c}\right)$.

Sinc function envelope of the pulse interference

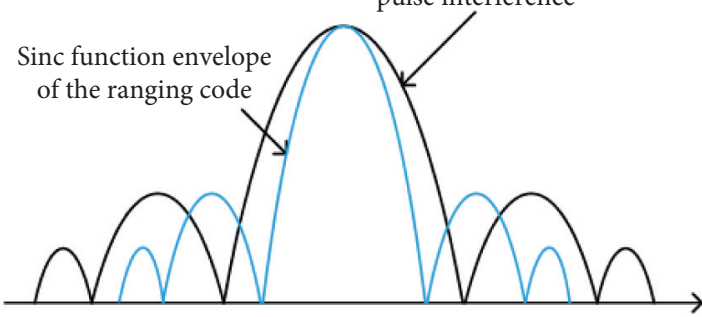

FIGURE 14: Sinc function envelope of pulse interference and ranging code spectrum line $\left(T_{p}=T_{c}\right)$.

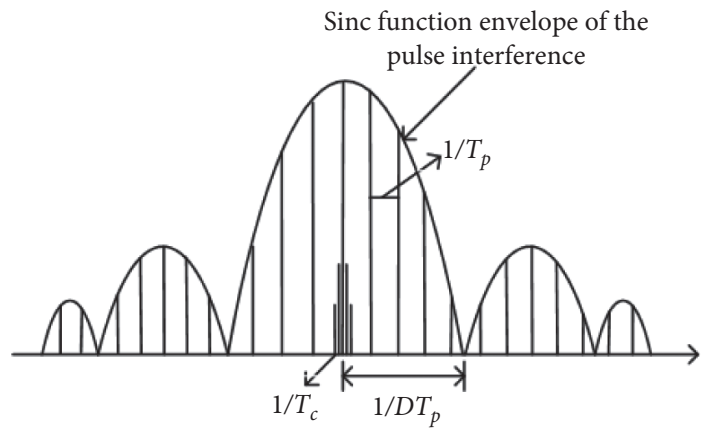

Figure 15: Sinc function envelope of pulse interference and ranging code spectrum line $\left(T_{p}<T_{c}\right)$.

interval, which means that most of the spectral lines in the main lobe of the pulse interference will affect the output power of the correlator. The discrete spectrum lines, which fall within the bandwidth of the correlation integrator, conform to $k=n-m$, so the output frequency domain signal of the correlator under this condition is obtained as follows:

$$
Z(f)=\sum_{m=-M}^{+M} T_{d} J_{n} P_{m} C_{n-m} \times \operatorname{sinc}\left(\left(f-\frac{m}{T_{c}}+\frac{m}{T_{p}}-\Delta f_{i}\right) T_{d}\right) e^{-j 2(n-m) \pi \tau / T_{c}}
$$

(3) $T_{p}<T_{c}$. Figure 15 is a schematic diagram of the pulse interference spectrum line and the ranging code spectrum line under the condition of $T_{p}<T_{c}$. In this case, only one pulse interference spectrum line falls within the ranging code envelope, which is the sine spectrum line with $m=0$, $n=k$ passing through the integrator, and the frequency domain expression of the output signal of the correlator at this time is,

$$
Z(f)=T_{d} J_{n} P_{0} C_{n} \times \operatorname{sinc}\left(\Delta f_{i} \cdot T_{d}\right) e^{-j 2 n \pi \tau / T_{c}} .
$$

Since only one spectral line can fall into the integrator band under the condition of $T_{p}<T_{c}$, the pulse interference at this time is similar to the single-frequency $\mathrm{CW}$ interference. The main lobe bandwidth of the pulse signal is relatively large, and the change of the duty cycle does not affect the number of pulse interference spectrum lines falling in the envelope of the ranging code. Therefore, the navigation 
signal is not sensitive to the change of the duty cycle when the repetition frequency is greater than $1 \mathrm{kHz}$, which meets the experiment results shown in Figure 11.

In summary, when the transmitted pulse interference power is constant, the pulse spectrum line is covered by the ranging code envelope under the condition of $T_{p}>T_{c}$. Almost all interference signals can enter the integrator together with the navigation signal for relevant processing. Therefore, in this case, the interference effect is the best. In the case of $T_{p}=T_{c}$, most of the pulse interference spectrum can enter the relevant processor, and the interference effect is worse than the condition of $T_{p}>T_{c}$. In the case of $T_{p}<T_{c}$, only one pulse interference spectrum falls within the envelope of the ranging code, and the interference effect is the worst. This can also explain the experimental phenomenon that the higher the repetition frequency under the same conditions, the higher the satellite equivalent $\mathrm{C} / \mathrm{N}_{0}$, and the higher the receiver sensitivity threshold.

\section{Conclusion}

In this paper, the time-frequency domain characteristics of the pulse interference signal were firstly analyzed as well as the influence on the despreading and demodulation performance of the BDS system. Then, we carried out the corresponding electromagnetic injection experiment on a certain BDS receiver. The following conclusions have been obtained:

(1) The existence of the pulse interference will cause an increase in the BER of the navigation system, and the BER is proportional to the duty cycle and the interference power.

(2) Under the condition that the frequency of the interference signal is $1561 \mathrm{MHz}$, the attenuation effect of the pulse interference on the $\mathrm{C} / \mathrm{N}_{0}$ of the navigation signal is significantly stronger than that of the single-frequency CW. This is because the pulse interference and the ranging code have similar spectral characteristics, which are correlation interference. The multiple discrete spectral lines falling in the band of the integrator can easily affect the despreading and demodulation of the navigation signal through the correlator, and a smaller interference power can achieve the same interference effect as a single-frequency $\mathrm{CW}$.

(3) When the duty cycle is constant, the performance of the pulse interference decreases with the increase of repetition frequency, and the receiver is more sensitive to low-repetition pulse interference. When the repetition frequency is constant, under the conditions of low repetition frequency $(60 \mathrm{~Hz}, 1 \mathrm{kHz})$, the duty cycle has a greater influence on the sensitivity threshold of the receiver, and the sensitivity threshold can differ by up to $15 \mathrm{~dB}$ under the same interference frequency. Under the condition of high repetition frequency $(1 \mathrm{MHz})$, the receiver is not sensitive to the duty cycle. The most sensitive frequency point of in-band pulse interference does not shift with the duty cycles. At this time, the pulse interference can be approximately equivalent to single-frequency $\mathrm{CW}$ interference.

\section{Data Availability}

No data were used to support this study.

\section{Conflicts of Interest}

The authors declare that there are no conflicts of interest regarding the publication of this paper.

\section{References}

[1] J. Guo, E. Wang, and H. He, "Analysis and evaluation of BDS performance based on real measured data," in Proceedings of the 2019 IEEE 1st International Conference on Civil Aviation Safety and Information Technology (ICCASIT), pp. 525-529, Kunming, China, October, 2019.

[2] Q. Zhao, Y. Zhao, L. Dong, and J. He, "Research on the influence of B1I ranging code distortion on BDS navigation performance," in Proceedings of the 2020 IEEE 2nd International Conference on Civil Aviation Safety and Information Technology, Weihai, China, October, 2020.

[3] M. Sharawi, D. Akos, and D. Aloi, "GPS C/N/sub 0/estimation in the presence of interference and limited quantization levels," IEEE Transactions on Aerospace and Electronic Systems, vol. 43, no. 1, pp. 227-238, 2007.

[4] W. T. Li, Z. G. Huang, and H. Xiao, "Analysis of the influence of interference on the tracking performance of satellite navigation receivers," Electronic Design Engineering, vol. 27, no. 08, pp. 119-123, 2019.

[5] M. F. Karsi and W. C. Lindsey, "Effects of CW interference on phase-locked loop performance," IEEE Transactions on Communications, vol. 48, no. 5, pp. 886-896, 2000.

[6] L. Y. Ye, Z. Y. Fan, H. Q. Zhang, Y. Liu, W. J. Wu, and Y. H. Hu, "Analysis of GNSS signal code tracking accuracy under Gaussian interference," Computer Science, vol. 47, no. 1, pp. 245-251, 2020.

[7] M. Bek, s. Elgamel, and E. Shaheen, "Interference effect on the post-correlation carrier to noise ratio for GPS receiver," The International Conference on Electrical Engineering, vol. 9, pp. 1-15, 2014.

[8] M. K. Bek, E. M. Shaheen, and S. A. Elgamel, "Analysis of the global position system acquisition process in the presence of interference," IET Radar, Sonar \& Navigation, vol. 10, no. 5, pp. 850-861, 2016.

[9] M. Pini, E. Falletti, and M. Fantino, "Performance evaluation of C/NO estimators using a real time GNSS software receiver," in Proceedings of the 2008 IEEE 10th International Symposium on Spread Spectrum Techniques and Applications, pp. 32-36, Bologna, Italy, August, 2008.

[10] A. T. Balaei, A. G. Dempster, and J. Barnes, "A novel approach in detection and characterization of CW interference of GPS signal using receiver estimation of $\mathrm{C} / \mathrm{No}$," in Proceedings of the 2006 IEEE/ION Position, Location, and Navigation Symposium, pp. 1120-1126, Coronado, CA, USA, April 2006.

[11] M. K. Bek, E. M. Shaheen, and S. A. Elgamel, "Classification and mathematical expression of different interference signals on a GPS receiver," Navigation, vol. 62, no. 1, pp. 23-37, 2015.

[12] T. Q. Zhang and H. L. Chu, "Evaluation of the impact of continuous wave interference on Beidou civil B1I signal tracking performance," in Proceedings of the 5th China 
satellite navigation Academic Conference-S6 Beidou/GNSS Test and Evaluation Technology, Nanjing, China, 2014.

[13] B. Qu, J. Wei, Z. Tang, and T. Yan, "Analysis of combined effects of multipath and CW interference on coherent delay lock loop," Wireless Personal Communications, vol. 77, no. 3, pp. 2213-2233, 2014.

[14] P. Wang, Y. Wang, and Y. J. Morton, "Characterization and impact analysis of radio frequency interference for GNSS reflectometry," in Proceedings of the IGARSS 2020 - 2020 IEEE International Geoscience and Remote Sensing Symposium, pp. 6190-6193, Waikoloa, HI, USA, October, 2020.

[15] L. Musumeci and F. Dovis, "A comparison of transformeddomain techniques for pulsed interference removal on GNSS signals," in Proceedings of the 2012 International Conference on Localization and GNSS, pp. 1-6, Starnberg, Germany, June, 2012.

[16] L. Tao, H. Tan, Q. Liu, and C. Fang, "Performance comparison of radar receivers subject to pulsed interference," in Proceedings of the 2018 IEEE Asia-Pacific Conference on Antennas and Propagation, pp. 1-2, APCAP, Auckland, New Zealand, August, 2018.

[17] A. T. Balaei, A. G. Dempster, and L. L. Presti, "Characterization of the effects of CW and pulse CW interference on the GPS signal quality," IEEE Transactions on Aerospace and Electronic Systems, vol. 45, no. 4, pp. 1418-1431, 2009.

[18] R. H. Liu, T. Li, and P. Shang, "Simulation analysis of pulse jamming of UWB system," Ship Electronic Engineering, vol. 31, no. 10, pp. 69-72, 2011.

[19] M. Y. Ding, R. Xu, and J. Y. Liu, "Analysis of the impact of pulse interference on software receivers," Navigation, Positioning and Timing, vol. 4, no. 03, pp. 58-65, 2017.

[20] L. Loh-Ming and L. Milstein, "Rejection of pulsed CW interference in PN spread-spectrum systems using complex adaptive filters," IEEE Transactions on Communications, vol. 31, no. 1, pp. 10-20, 1983.

[21] Y. F. Ding and W. S. Li, "Performance analysis of pulse interference direct sequence spread spectrum system," Computer Simulation, vol. 27, no. 05, pp. 9-12+115, 2010.

[22] X. Dai, J. Nie, B. Li, Z. Lu, and G. Ou, "Performance of GNSS receivers with AGC in noise pulse interference," in Proceedings of the 20165 th International Conference on Computer Science and Network Technology (ICCSNT), pp. 735-740, Changchun, China, December, 2016.

[23] Q. Zhang, Y. Chen, and E. Cheng, "Experimental research on the effect of ESD on Beidou navigation receiver," in Proceedings of the 2019 IEEE 6th International Symposium on Electromagnetic Compatibility (ISEMC), IEEE, Nanjing, China, November, 2019.

[24] D. Zhang, Y. Chen, and E. Cheng, "Experimental research on continuous-wave electromagnetic radiation effects of a certain unmanned target drone," Journal of Hebei Normal University (Philosophy and Social Sciences Edition), vol. 41, no. 1, pp. 39-44, 2017.

[25] Q. Zhang, Y. Wang, and E. Cheng, "Effect law of navigation receiver tracking loop under electromagnetic interference," Transactions of Beijing Institute of Technology, vol. 41, no. 2, pp. 207-213, 2021.

[26] Q. Zhang, Y. Wang, and E. Cheng, "Investigation on the effect law and prediction method of out-of-band electromagnetic interference in navigation receiver," Systems Engineering and Electronics, vol. 43, no. 9, pp. 2588-2593, 2021.

[27] Y. Fan, E. Cheng, M. Wei, Q. Zhang, and Y. Chen, "Effects of $\mathrm{CW}$ interference on the BDS receiver and analysis on the coupling path of electromagnetic energy," IEEE Access, vol. 7, pp. 155885-155893, 2019.

[28] Q. Zhang, Y. Wang, and E. Cheng, "Research on the electromagnetic interference effect of UAV satellite navigation system," Systems Engineering and Electronics, vol. 42, no. 12, pp. 2684-2691, 2020.

[29] S. S. Tan, J. Yang, and D. X. Ming, Theory and application of complex electromagnetic environment simulation test system for satellite navigation terminal, Science Press, Beijing, 2018.

[30] Y. Q. Liu, X. L. Hu, and Y. H. Ran, "Research on anti-singlefrequency interference performance of satellite navigation signals," Chinese Journal of Electronics, vol. 39, no. 6, pp. 1410-1416, 2011.

[31] Q. Zhang, W. Yuming, and E. Cheng, "Investigation on inband interference effect and out-of-band interference mechanism of B1I navigation receiver," Radioengineering, vol. 30, no. 3, 2021.

[32] S. G. Mohinder, P. A. Angus, and G. B. Chris, "GNSS signal structure, characteristics, and information utilization," Global Navigation Satellite Systems, Inertial Navigation, and Integration, Wiley, Hoboken, NJ, USA, pp. 93-143, 2020.

[33] L. He, P. Zeng, H. Zhou, and Y. Wen, "Improved antenna phase center offsets for BDS-2 IGSO/MEO satellites based on MGEX long-term observations," IEEE Access, vol. 8, pp. 195844-195856, 2020.

[34] J. Jang, M. Paonni, and B. Eissfeller, "CW interference effects on tracking performance of GNSS receivers," IEEE Transactions on Aerospace and Electronic Systems, vol. 48, no. 1, pp. 243-258, 2012. 